

В. А. Горелов, О структуре множества $E$-функций, удовлетворяющих линейным дифференциальным уравнениям второго порядка, Матем. заметки, 2005, том 78, выпуск $3,331-348$

DOI: https://doi.org/10.4213/mzm2591

Использование Общероссийского математического портала Math-Net.Ru подразумевает, что вы прочитали и согласны с пользовательским соглашением http://www . mathnet.ru/rus/agreement

Параметры загрузки:

IP : 54.92 .164 .108

26 апреля 2023 г., $17: 28: 12$






\title{
О СТРУКТУРЕ МНОЖЕСТВА Е-ФУНКЦИЙ, УДОВЛЕТВОРЯЮЩИХ ЛИНЕЙНЫМ ДИФФЕРЕНЦИАЛЬНЫМ УРАВНЕНИЯМ ВТОРОГО ПОРЯДКА
}

\author{
В. А. Горелов
}

Доказьвается частный с лучай гипотезы Зигеля о представимости $E$-функций в виде многочлена от гипергеометрических функций. Доказьваются некоторые утверждения о трансцендентности и линейной независимости значений $E$-функций, ранее сформулированные А. Б. Шидловским.

Библиографоия: 10 названий.

Введение. В 1929 г. К. Зигель опубликовал статью [1], где излагался метод, позволяющий исследовать арифметическую природу значений одного класса целых аналитических функций, так назьваемых $E$-функций, удовлетворяющих системам линейных дифференциальных уравнений. Современное состояние метода и историю вопроса можно найти в книге [2].

Зигель высказал гипотезу, что всякая $E$-функция, удовлетворяющая линейному дифференциальному уравнению с коэффищиентами из $\mathbb{C}(z)$, представляется в виде многочлена с алгебраическими коэффициентами от $z$ и конечного числа гипергеометрических $E$-функций, а также функций, получающихся из них заменой $z$ на $\alpha z$ при $\alpha \in \mathbb{A}$, где $\mathbb{A}-$ множество всех алгебраических чисел.

Гипергеометрическими $E$-функциями, например, являются используемые в настоящей работе функции

$$
\varphi_{\lambda}(z)=1+\sum_{n=1}^{\infty} \frac{z^{n}}{(\lambda+1) \cdots(\lambda+n)}, \quad \lambda \in \mathbb{Q}, \quad \lambda \neq-1,-2, \ldots,
$$

и $e^{z}=\varphi_{0}(z)$.

В работах автора [3], [4] была выяснена структура множества $E$-функций, удовлетворяющих линейным дифференциальным уравнениям 1-го порядка и линейным однородным дифференциальным уравнениям 2-го порядка соответственно. Гипотеза Зигеля для этих случаев оказалась справедливой.

В настояшей работе рассматриваются $E$-функции, удовлетворяюшие уравнениям

$$
Q_{2} y^{\prime \prime}+Q_{1} y^{\prime}+Q_{0} y=Q, \quad Q_{2}, Q_{1}, Q_{0}, Q \in \mathbb{C}[z] \text {. }
$$

Доказываются следующие теоремы. 
ТЕОрема 1. Функиия $f(z)$ тогда и только тогда является Е-функиией, удовлетворяющей уравнению (1), когда

$$
f(z)=P_{0} \varphi_{\lambda}(\alpha z)+P_{1} \varphi_{\lambda_{1}}\left(\alpha_{1} z\right)+P
$$

либо

$$
f(z)=P_{0} f_{1}(z)+P_{1} f_{1}^{\prime}(z)+P,
$$

где $P_{0}, P_{1}, P \in \mathbb{A}[z], \lambda, \lambda_{1} \in \mathbb{Q}, \alpha, \alpha_{1} \in \mathbb{A}, f_{1}(z)$ - Е-функиия, удовлетворяющая уравнению

$$
y^{\prime \prime}+\left(a+\frac{a_{1}}{z}\right) y^{\prime}+\left(b+\frac{b_{1}}{z}+\frac{b_{2}}{z^{2}}\right) y=c+\frac{c_{1}}{z},
$$

$a, a_{1}, b, b_{1}, b_{2}, c, c_{1} \in \mathbb{A}$.

ТЕорема 2. Функиия $f(z)$ тогда и только тогда является Е-функиией, удовлетворяющей уравнению (1) и алгебраически зависимой $c f^{\prime}(z)$ над $\mathbb{C}(z)$, когда

$$
f(z)=P_{0} \varphi_{k}(\alpha z)+P_{1} \varphi_{k}(\sigma \alpha z)+P
$$

либо

$$
f(z)=P_{0} \varphi_{\lambda}^{2}(\alpha z)+P_{1} \varphi_{\lambda}(\alpha z)+P,
$$

әде $P_{0}, P_{1}, P \in \mathbb{A}[z], k \in \mathbb{Z}^{+}, \lambda, \sigma \in \mathbb{Q}, \alpha \in \mathbb{A}$.

СлЕДСТВИЕ. Если алгебраически зависимые над $\mathbb{C}(z)$ E-функиии $f_{1}(z), f_{2}(z)$ составляют решение системы линейных дифференциальных уравнений 1-го порядка с коэффициентами из $\mathbb{C}(z)$, то обе они имеют вид (5) или (6).

ТЕорема 3. Если Е-функиия $f(z)$ удовлетворяет уравнению (1) и линейно независима с $f^{\prime}(z)$ и 1 над $\mathbb{C}(z)$, то числа $f(\xi), f^{\prime}(\xi)$ и 1 линейно независимь над $\mathbb{A}$ при любом $\xi \in \mathbb{A}, \xi Q_{2}(\xi) \neq 0$.

Теорема 3 является частным случаем утверждения, высказанного Шидловским [5, гипотеза А]. Из теоремы 3 следует

ТЕОремА 4. Если Е-функиия $f(z)$ удовлетворяет уравнению (1) и не удовлетворяет никакому линейному дифференциальному уравнению 1-го порядка с коәффичиентами из $\mathbb{C}(z)$, то числа $f(\xi)$ и $f^{\prime}(\xi)$ трансиендентны при любом $\xi \in \mathbb{A}$, $\xi Q_{2}(\xi) \neq 0$.

Ранее теорема 4 в виде гипотезы также высказьвалась Шидловским [2, гл. 6, 1 1]. Условие $Q_{2}(\xi) \neq 0$ в теореме 4 не является необходимым. Например, $E$-функция

$$
f(z)=e^{2 z}+z e^{z}+1
$$

удовлетворяет уравнениям $y^{\prime}=2 y-(z-1) e^{z}-2$ и $(z-1) y^{\prime \prime}-(3 z-2) y^{\prime}+2 z y=2 z$. Числа $f(1)$ и $f^{\prime}(1)$ трансцендентны, хотя $Q_{2}(1)=0$. 


\section{1. Вспомогательные утверждения.}

ЛЕмма 1. Пусть Е-функиия $f(z)$ удовлетворяет линейному дифференциальному уравнению

$$
Q_{m} y^{(m)}+Q_{m-1} y^{(m-1)}+\cdots+Q_{0} y=Q, \quad m \geqslant 1,
$$

с коэффициентами из $\mathbb{C}[z]$, взаимно простыми в совокупности, и не удовлетворяет никакому линейному (или, при $Q \equiv 0,-$ линейному однородному) дифференииальному уравнению меньшего порядка с коэффиииентами из $\mathbb{C}[z]$. Тогда коэффиииенты уравнения (7) определены однозначно с точностью до множителя из $\mathbb{C}$ и могут быть выбраны из $\mathbb{A}[z]$.

Лемма 1 следует из результатов книги [2, гл. $3, \S 2]$.

Определение используемых далее понятий регулярной особой точки и показателей однородного дифференциального уравнения см., например, в книгах [6] или [7].

ЛЕмма 2 (см. [4, леммы 4-6]). При условиях леммы 1 всякая особая точка $\xi \neq 0$ дифференииального уравнения (7) есть регулярная особая точка соответствующего ему однородного уравнения с иелыми неотрииательными показателями $r_{1}<r_{2}<\cdots<r_{m}$, а всякое решение уравнения (7), как и соответствующего однородного уравнения, голоморфно в точке $\xi$.

Лемма 3 (см. [4, лемма 7]). Если при условиях леммы $1 Q_{m}(\xi)=0, \xi \neq 0$, то

$$
\frac{Q_{k}}{Q_{m}}=\frac{c_{k}}{(z-\xi)^{m-k}}+\frac{\psi_{k}(z)}{(z-\xi)^{m-k-1}}, \quad k=0,1, \ldots, m-1,
$$

где $c_{k} \in \mathbb{Z},-c_{m-1} \in \mathbb{N},(-1)^{m} c_{0}=r_{1} \cdots r_{m} \in \mathbb{Z}^{+}, \psi_{k}(z) \in \mathbb{A}(z), \psi_{k}(\xi) \neq \infty$.

Лемма 4. Если при условиях леммы $3 c_{0} \neq 0$, то для некоторого $P \in \mathbb{A}[z]$ функиия $g(z)=(f(z)-P) /(z-\xi)^{r_{1}}$ является E-функиией.

Доказательство аналогично доказательству леммы 9 статьи [4] с той лишь разницей, что ввиду отсутствия утверждения $\operatorname{ord}_{z=\xi}(f(z)-P)=r_{1}$ использование леммы 8 статьи [4] уже не требуется.

ЛЕмМа 5. Если при условиях леммы 1 точка $\xi=0$ есть особая точка уравнения (7), то она является регулярной особой точкой соответствующего ему однородного дифференииального уравнения.

ДокАЗАТЕЛьство. В случае $m=1$ утверждение леммы следует из определения $E$-функции (см. [3, лемма 10$])$. В случае $Q \equiv 0$ лемма справедлива согласно доказанному Андрэ [8, следствие 4.4]. Пусть $Q \not \equiv 0$. Продифференцировав уравнение (7), получим равенство

$$
Q_{m} y^{(m+1)}+\left(Q_{m}^{\prime}+Q_{m-1}\right) y^{(m)}+\cdots+\left(Q_{1}^{\prime}+Q_{0}\right) y^{\prime}+Q_{0}^{\prime} y=Q^{\prime} .
$$

Умножая это равенство на $Q$ и вычитая из него равенство $(7)$, умноженное на $Q^{\prime}$, получаем

$$
Q_{m} Q y^{(m+1)}+\left(Q\left(Q_{m}^{\prime}+Q_{m-1}\right)-Q_{m} Q^{\prime}\right) y^{(m)}+\cdots+\left(Q Q_{0}^{\prime}-Q_{0} Q^{\prime}\right) y=0
$$


откуда

$$
\begin{aligned}
y^{(m+1)} & +\left(\frac{Q_{m-1}}{Q_{m}}+\frac{Q_{m}^{\prime}}{Q_{m}}-\frac{Q^{\prime}}{Q}\right) y^{(m)}+\left(\frac{Q_{m-2}}{Q_{m}}+\frac{Q_{m-1}^{\prime}}{Q_{m}}-\frac{Q_{m-1}}{Q_{m}} \frac{Q^{\prime}}{Q}\right) y^{(m-1)}+\cdots \\
& +\left(\frac{Q_{0}}{Q_{m}}+\frac{Q_{1}^{\prime}}{Q_{m}}-\frac{Q_{1}}{Q_{m}} \frac{Q^{\prime}}{Q}\right) y^{\prime}+\left(\frac{Q_{0}^{\prime}}{Q_{m}}-\frac{Q_{0}}{Q_{m}} \frac{Q^{\prime}}{Q}\right) y=0 .
\end{aligned}
$$

Полученное уравнение является однородным дифференциальным уравнением наименьшего порядка, которому удовлетворяет $E$-функция $f(z)$. Поэтому точка $z=0$ является его регулярной особой точкой. Но это возможно только в случае, когда порядки полюсов функций $Q_{m-1} / Q_{m}, Q_{m-2} / Q_{m}, \ldots, Q_{0} / Q_{m}$ в точке $z=0$ не превосходят соответственно $1,2, \ldots, m$. Лемма доказана.

ЛЕмма 6. Пусть дифференииальные операторы $F_{1}, F_{2} \in \mathbb{A}[z, d / d z]$ таковы, что

$$
\begin{gathered}
F_{1}=P_{n}\left(\frac{d}{d z}\right)^{n}+\cdots+P_{1} \frac{d}{d z}+P_{0}, \quad P_{j} \in \mathbb{A}[z], \quad j=0, \ldots, n \\
F_{2}=Q_{m}\left(\frac{d}{d z}\right)^{m}+\cdots+Q_{1} \frac{d}{d z}+Q_{0}, \quad Q_{i} \in \mathbb{A}[z], \quad i=0, \ldots, m \\
F_{1} F_{2}=R_{n+m}\left(\frac{d}{d z}\right)^{n+m}+\cdots+R_{1} \frac{d}{d z}+R_{0}, \quad P_{n} Q_{m} \not \equiv 0 \\
\operatorname{deg} R_{n+m}=\max _{0 \leqslant k \leqslant n+m} \operatorname{deg} R_{k} .
\end{gathered}
$$

Tогда

$$
\operatorname{deg} Q_{m}=\max _{0 \leqslant i \leqslant m} \operatorname{deg} Q_{i}
$$

ДокАЗАТЕльство. Пусть лемма неверна, а $\theta$ - наибольший индекс, для которого

$$
\operatorname{deg} Q_{\theta}=\max _{0 \leqslant i<m} \operatorname{deg} Q_{i}>\operatorname{deg} Q_{m}
$$

Обозначим $\sigma$ наибольший из индексов таких, что

$$
\operatorname{deg} P_{\sigma}=\max _{0 \leqslant j \leqslant n} \operatorname{deg} P_{j}, \quad 0 \leqslant \sigma \leqslant n .
$$

Тогда $R_{n+m}=P_{n} Q_{m}, \mathrm{a}$

$$
R_{\sigma+\theta}=P_{\sigma} Q_{\theta}+\sum_{j=1}^{n-\sigma}\left(\begin{array}{c}
\sigma+j \\
j
\end{array}\right) P_{\sigma+j} Q_{\theta}^{(j)}+\sum_{-\theta \leqslant i \leqslant m-\theta} \sum_{\substack{j \neq 0 \\
i \neq 0}}^{n-\sigma+i}\left(\begin{array}{c}
\sigma-i+j \\
j
\end{array}\right) P_{\sigma-i+j} Q_{\theta+i}^{(j)}
$$

Следовательно, $\operatorname{deg} R_{\sigma+\theta}=\operatorname{deg} P_{\sigma} Q_{\theta}>\operatorname{deg} R_{n+m}$. Ввиду полученного противоречия лемма доказана.

ЛЕмма 7. При условиях леммы 1 справедливо равенство

$$
\operatorname{deg} Q_{m}=\max _{0 \leqslant i<m} \operatorname{deg} Q_{i}
$$


ДокАЗАТЕЛЬСтво. Прежде всего заметим, что если в уравнении (7) $Q \not \equiv 0$, то утверждение леммы вьполняется или не выполняется для уравнений (7) и (8) одновременно.

Известно, что голоморфная в точке $z=0$ функция $F(z)=\sum_{\nu=0}^{\infty} a_{\nu} z^{\nu}$ удовлетворяет уравнению

$$
Q_{m} y^{(m)}+Q_{m-1} y^{(m-1)}+\cdots+Q_{0} y=0, \quad Q_{m}, \ldots, Q_{0} \in \mathbb{A}[z]
$$

тогда и только тогда, когда последовательность $\left\{a_{\nu}\right\}$ удовлетворяет линейному разностному уравнению

$$
p_{0} a_{\nu}+p_{1} a_{\nu-1}+\cdots+p_{n} a_{\nu-n}=0, \quad p_{0}, \ldots, p_{n} \in \mathbb{A}[\nu], \quad \nu=0,1, \ldots,
$$

причем $\max _{0 \leqslant i \leqslant n} \operatorname{deg} p_{i}=m$ (см., например, $[9$, с. 142,143$\left.]\right)$. Положим в уравнении $(9)$

$$
Q_{k}=q_{k, 0}+q_{k, 1} z+\cdots+q_{k, \mu} z^{\mu}, \quad q_{k, i} \in \mathbb{A}, \quad \mu=\max _{0 \leqslant k \leqslant m} \operatorname{deg} Q_{k} .
$$

Пусть $s_{0}$ и $s_{1}$ - наименьшее (соответственно наибольшее) из чисел $s$ таких, что

$$
\sum_{j=0}^{m}\left|q_{m-j, s-j}\right| \neq 0 .
$$

Можно считать, что $0 \leqslant s_{0} \leqslant \min (m, \mu) \leqslant s_{1} \leqslant m+\mu$. Как установлено при доказательстве леммы 3 статьи [4], в уравнении (10) $n=s_{1}-s_{0}$,

$$
\begin{aligned}
p_{0}= & \sum_{j=0}^{\min \left\{s_{0}, m-1\right\}} q_{m-j, s_{0}-j} \nu(\nu-1) \cdots(\nu-m+j+1)+q_{0, s_{0}-m}, \\
p_{n}= & \sum_{j=0}^{\min \left\{s_{1}, m-1\right\}} q_{m-j, s_{1}-j}(\nu-n) \cdots(\nu-n-m+j+1)+q_{0, s_{1}-m}, \\
& \operatorname{deg} p_{i}=\max _{j}\left\{m-j \mid q_{m-j, s_{0}+i-j} \neq 0\right\}, \quad i=0,1, \ldots, n .
\end{aligned}
$$

Всякой $G$-функции (определение см. в [2]) $F(z)=\sum_{\nu=0}^{\infty} a_{\nu} z^{\nu}$ можно взаимно однозначно сопоставить $E$-функцию $f(z)=\sum_{\nu=0}^{\infty}\left(a_{\nu} / \nu !\right) z^{\nu}$. Если последовательность $\left\{a_{\nu}\right\}$ удовлетворяет уравнению (10), то последовательность $\left\{a_{\nu} / \nu !\right\}$ удовлетворяет, очевидно, уравнению

$$
\omega_{0} \frac{a_{\nu}}{\nu !}+\omega_{1} \frac{a_{\nu-1}}{(\nu-1) !}+\cdots+\omega_{n} \frac{a_{\nu-n}}{(\nu-n) !}=0
$$

где $\omega_{0}=\nu(\nu-1) \cdots(\nu-n+1) p_{0}, \ldots, \omega_{n}=p_{n}, \operatorname{deg} \omega_{i} \leqslant m+n-i, i=0,1, \ldots, n$.

Следовательно, $G$-функция $F(z)$ удовлетворяет уравнению (9) тогда и только тогда, когда соответствующая ей $E$-функция $f(z)$ удовлетворяет аналогичному дифференциальному уравнению, причем порядки этих уравнений отличаются не более, чем на $n$.

Как установлено в статье Андрэ $[8, \S 3]$, если $G$-функция $F(z)$ удовлетворяет уравнению $(9)$, где $m$ - наименьшее из возможных, то точка $z=\infty$ является регулярной особой 
точкой уравнения (9). Это равносильно условию (см. [6, гл. 5, п. 18.3]) $\operatorname{deg} Q_{k}=k+k_{0}$, $k=0,1, \ldots, m, k_{0}=\operatorname{deg} Q_{0}$, откуда $s_{1}=m+k_{0}=\mu, q_{m, s_{1}} \neq 0$.

Кроме того, если точка $z=0$ неособая, то $q_{m, 0} \neq 0, s_{0}=0$, а если особая, то ввиду ее регулярности (см. $[8, \S 3])$ имеем $s_{0}=\operatorname{ord}_{z=0} Q_{m}, q_{m, s_{0}} \neq 0$.

Поскольку $q_{m, s_{0}} \neq 0, q_{m, s_{1}} \neq 0$, то $\operatorname{deg} \omega_{0}=m+n$, а разностному уравнению $(12)$ соответствует дифференциальное уравнение

$$
R_{m+n} y^{(m+n)}+\cdots+R_{1} y^{\prime}+R_{0} y=0, \quad R_{m+n}, \ldots, R_{0} \in \mathbb{A}[z] .
$$

Из неравенств $\operatorname{deg} \omega_{i} \leqslant m+n-i$, равенств $\operatorname{deg} \omega_{0}=m+n$ и (11) следует, что

$$
R_{m+n}=q_{m, s_{0}} z^{s_{1}}, \quad \operatorname{deg} R_{i} \leqslant s_{1}, \quad i=0,1, \ldots, m+n .
$$

Уравнение (13), которому удовлетворяет $E$-функция $f(z)$, можно представить в виде $F_{3} y=0$, где $F_{3} \in \mathbb{A}[z, d / d z]$. Пусть $F_{2} \in \mathbb{A}[z, d / d z]$ - дифференциальньй оператор наименьшего порядка, аннулируюший функцию $f(z)$. Тогда $F_{3}=F_{1} F_{2}$, где $F_{1}-$ дифференциальньй оператор с коэффициентами из $\mathbb{A}(z)$ (см. [7, гл. 5, п. 5.4]). Согласно лемме 6 степень многочлена, являюшегося старшим коэффициентом в $F_{2}$, больше или равна максимуму степеней остальных коэффициентов. Из теории Вимана-Валирона следует (см., например, $[10, \S 75]$ ), что это неравенство не может быть строгим. Отсюда ввиду замечания в начале доказательства получаем утверждение леммы.

Заметим, что если из условий леммы 7 убрать требование минимальности числа $m$, то утверждение леммы становится неверньм. Например, $E$-функция $\varphi_{\lambda}(z)$ удовлетворяет уравнению $z y^{\prime \prime}+\left(z^{2}-z+\lambda+1\right) y^{\prime}+\left(-z^{2}+\lambda z-1\right) y=\lambda z$.

ЛЕмма 8 (см., например, [7, с. 526]). Пусть в дифференииальном уравнении (1)

$$
\frac{Q_{1}}{Q_{2}}=\frac{1-c_{1}}{z-\xi}+\psi_{1}(z), \quad \frac{Q_{0}}{Q_{2}}=\frac{c_{0}}{(z-\xi)^{2}}+\frac{\psi_{0}(z)}{z-\xi}
$$

где $\xi \in \mathbb{C}, \psi_{1}(z), \psi_{0}(z)-\oint у н к и и и$, голоморфные в точке $z=\xi$. Тогда сумма $и$ произведение показателей однородного дифференциального уравнения, соответствующего (1), в точке $\xi$ равны соответственно $c_{1} и c_{0}$.

Следующие три леммы доказьваются непосредственньм вычислением.

Лемма 9. Пусть $f, Q_{0}, Q_{1}, Q, P, P_{1}$ - произвольные аналитические функиии, причем функиия $f$ удовлетворяет дифференциальному уравнению

$$
y^{\prime \prime}+Q_{1} y^{\prime}+Q_{0} y=Q
$$

Тогда функиия $g=P f$ удовлетворяет уравнению

$$
y^{\prime \prime}+\left(Q_{1}-\frac{2 P^{\prime}}{P}\right) y^{\prime}+\left(Q_{0}-\frac{P^{\prime \prime}}{P}-\left(Q_{1}-\frac{2 P^{\prime}}{P}\right) \frac{P^{\prime}}{P}\right) y=P Q
$$

а функиия $g_{1}=f+P_{1}-$ уравнению

$$
y^{\prime \prime}+Q_{1} y^{\prime}+Q_{0} y=Q+P_{1}^{\prime \prime}+P_{1}^{\prime} Q_{1}+P_{1} Q_{0} .
$$


СлЕДСТВИЕ. При условиях леммы 9 функиия $g=e^{\alpha z} f$, где $\alpha \in \mathbb{C}$, удовлетворяет дифференииальному уравнению

$$
y^{\prime \prime}+\left(Q_{1}-2 \alpha\right) y^{\prime}+\left(Q_{0}-\alpha Q_{1}+\alpha^{2}\right) y=e^{\alpha z} Q .
$$

Лемма 10. Пусть $f, Q_{0}, Q_{1}, Q_{2}, Q$ - произвольные аналитические функиии, причем $Q_{0} Q_{2} \not \equiv 0$, а функиия $f$ удовлетворяет дифференциальному уравнению

$$
y^{\prime \prime}+\frac{Q_{1}}{Q_{2}} y^{\prime}+\frac{Q_{0}}{Q_{2}} y=\frac{Q}{Q_{2}} .
$$

Тогда функиия $g=f^{\prime}$ удовлетворяет дифференциальному уравнению

$$
y^{\prime \prime}+\left(\frac{Q_{1}}{Q_{2}}+\frac{Q_{2}^{\prime}}{Q_{2}}-\frac{Q_{0}^{\prime}}{Q_{0}}\right) y^{\prime}+\left(\frac{Q_{0}}{Q_{2}}+\frac{Q_{1}^{\prime}}{Q_{2}}-\frac{Q_{0}^{\prime}}{Q_{0}} \frac{Q_{1}}{Q_{2}}\right) y=\frac{Q^{\prime}}{Q_{2}}-\frac{Q_{0}^{\prime}}{Q_{0}} \frac{Q}{Q_{2}} .
$$

Лемма 11. Пусть $f, R_{1}, R_{2}, P_{1}, P_{2}$ - произвольные аналитические функиии, причем функиия $f$ удовлетворяет дифференииальному уравнению

$$
y^{\prime}=R_{1} y+R_{2} \text {. }
$$

Тогда функиия $g=P_{1} f+P_{2}$ удовлетворяет дифференииальному уравнению

$$
y^{\prime}=\left(R_{1}+\frac{P_{1}^{\prime}}{P_{1}}\right) y-\left(R_{1}+\frac{P_{1}^{\prime}}{P_{1}}\right) P_{2}+P_{1} R_{2}+P_{2}^{\prime} .
$$

ЛЕмма 12. Пусть Е-функиия $f$ удовлетворяет дифференциальному уравнению

$$
y^{\prime}=\left(a_{0}+\frac{a_{1}}{z}\right) y+a_{2} z^{k} \varphi_{\lambda}(\alpha z)+R,
$$

$a_{0}, a_{1}, a_{2}, \alpha \in \mathbb{A}, \lambda \in \mathbb{Q}, k \in \mathbb{Z}, R \in \mathbb{A}(z)$. Тогда $f=z^{n} g+P$, где $P \in \mathbb{A}[z], n \in \mathbb{Z}^{+}, a$ $g$ - E-функиия, удовлетворяющая дифференииальному уравнению вида (4).

ДокАЗАТЕЛЬСтво. Продифференцировав равенство (14), получим

$$
y^{\prime \prime}=\left(a_{0}+\frac{a_{1}}{z}\right) y^{\prime}-\frac{a_{1}}{z^{2}} y+a_{2} z^{k}\left(\alpha+\frac{k-\lambda}{z}\right) \varphi_{\lambda}(\alpha z)+R^{\prime}+a_{2} \lambda z^{k-1} .
$$

Вычитая из этого равенства равенство (14), умноженное на $\alpha+(k-\lambda) / z$, получим

$$
y^{\prime \prime}+\left(a_{3}+\frac{a_{4}}{z}\right) y^{\prime}+\left(a_{5}+\frac{a_{6}}{z}+\frac{a_{7}}{z^{2}}\right) y=P_{1}+\frac{a_{8}}{z}+\frac{a_{9}}{z^{2}},
$$

где $a_{3}, \ldots, a_{9} \in \mathbb{A}$, а $P_{1}$, как легко видеть, можно выбрать из $\mathbb{A}[z]$. Пусть $\operatorname{deg} P_{1}=n>0$. Рассмотрим многочлен $P_{2} \in \mathbb{A}[z], \operatorname{deg} P_{2} \leqslant n-1$, такой, что функция $g=\left(f-P_{2}\right) / z^{n}$ является целой и, следовательно, $E$-функцией. Согласно лемме 9 функция $f-P_{2}$ удовлетворяет уравнению

$$
y^{\prime \prime}+\left(a_{3}+\frac{a_{4}}{z}\right) y^{\prime}+\left(a_{5}+\frac{a_{6}}{z}+\frac{a_{7}}{z^{2}}\right) y=P_{3}+\frac{a_{10}}{z}+\frac{a_{11}}{z^{2}},
$$

где $a_{10}, a_{11} \in \mathbb{A}, P_{3} \in \mathbb{A}[z], \operatorname{deg} P_{3} \leqslant n$, а функция $g$ - уравнению вида $(15)$, где числа $a_{3}, \ldots, a_{7}$, вообще говоря, изменятся, а правая часть поделится на $z^{n}$. Поскольку функция $g$ целая, то правая часть этого нового уравнения должна иметь вид $a_{12}+a_{13} / z+$ $a_{14} / z^{2}$. В случае $a_{7}=0$ имеем также $a_{14}=0$, и лемма справедлива. Если $a_{7} \neq 0$, то, рассмотрев функцию $g+a_{14} / a_{7}$, получим, что она удовлетворяет уравнению (4). Лемма доказана. 
Лемма 13. Пусть Е-функиия

$$
f=R_{0} f_{1}+R_{1} f_{1}^{\prime}+R, \quad R_{0}, R_{1}, R \in \mathbb{A}(z),
$$

а $f_{1}-$-фуункиия, удовлетворяющая уравнению

$$
y^{\prime}=\left(a_{0}+\frac{a_{1}}{z}\right) y+\left(P+\frac{a}{z}\right) \varphi_{\lambda}(\alpha z)+R_{2}
$$

где $a_{0}, a_{1}, a, \alpha \in \mathbb{A}, \lambda \in \mathbb{Q}, P \in \mathbb{A}[z], R_{2} \in \mathbb{A}(z)$. Тогда

$$
f=R_{3} \varphi_{\lambda}(\alpha z)+R_{4} \varphi_{\lambda_{1}}\left(\alpha_{1} z\right)+R_{5}, \quad \lambda_{1} \in \mathbb{Q}, \quad \alpha_{1} \in \mathbb{A}
$$

либо

$$
f=R_{3} g+R_{4} g^{\prime}+R_{5}, \quad R_{3}, R_{4}, R_{5} \in \mathbb{A}(z),
$$

где $g-$-функиия, удовлетворяющая уравнению вида (4).

ДокАЗАТЕЛЬСтво. Из уравнений (16) и (17) получаем

$$
f=R_{6} f_{1}+R_{7} \varphi_{\lambda}(\alpha z)+R_{8}, \quad R_{6}, R_{7}, R_{8} \in \mathbb{A}(z) .
$$

Функция $f_{1}+c z^{k} \varphi_{\lambda}(\alpha z), k \in \mathbb{Z}^{+}, c \in \mathbb{A}$, как следует из уравнения (17) и леммы 11 , удовлетворяет уравнению

$y^{\prime}=\left(a_{0}+\frac{a_{1}}{z}\right) y+\left(P+\left(\alpha-a_{0}\right) c z^{k}+\left(k-\lambda-a_{1}\right) c z^{k-1}+\frac{a}{z}\right) \varphi_{\lambda}(\alpha z)+R_{2}+\lambda c z^{k-1}$.

Отсюда видно, что в случае $\alpha-a_{0} \neq 0$ и в случае $\alpha-a_{0}=0, k-\lambda-a_{1} \neq 0, k=1,2, \ldots$, можно подобрать $P_{1}=\sum c_{k} z^{k} \in \mathbb{A}[z]$ так, чтобы функция $f_{2}=f_{1}+P_{1} \varphi_{\lambda}(\alpha z)$ удовлетворяла уравнению вида (17), но уже с $P \equiv 0$. В случае $\alpha-a_{0}=0, k_{0}-\lambda-a_{1}=0$, $k_{0} \in \mathbb{N}$, очевидно, что при правильном выборе $P_{1}$ можно обнулить все одночлены в многочлене $P$, кроме, быть может, одного, содержащего $z^{k_{0}-1}$, а также дробь $a / z$. Таким образом, во всех случаях функция $f_{2}$ удовлетворяет уравнению (14), где $k \geqslant-1$. Если в равенстве (14) $a_{2} \neq 0$, то из определения функции $f_{2}$, равенств (20) и (14) получим $f=R_{9} f_{2}+R_{10} f_{2}^{\prime}+R_{11}$, где $R_{9}, R_{10}, R_{11} \in \mathbb{A}(z)$. Согласно лемме 12 для некоторых $n \in \mathbb{Z}^{+}$и $P_{2} \in \mathbb{A}[z]$ справедливо равенство $f_{2}=z^{n} g+P_{2}$, где $g-E$-функция, удовлетворяюшая дифференциальному уравнению (4). Следовательно, функция $f$ имеет вид (19), что и требовалось доказать. Если $a_{2}=0$, то согласно теореме 2 работы [3] $f_{2}=P_{3} \varphi_{\lambda_{1}}\left(\alpha_{1} z\right)+P_{4}, \lambda_{1} \in \mathbb{Q}, \alpha_{1} \in \mathbb{A}, P_{3}, P_{4} \in \mathbb{A}[z]$. Выразив $f_{1}$ через $f_{2}$ и подставив результат в (20), получим равенство (18). Лемма доказана.

\section{2. Основная лемма.}

ЛЕмма 14. Если Е-функиия $f(z)$ удовлетворяет уравнению (1), то она имеет вид либо (18), либо (16), где $f_{1}(z)-E$-функция, удовлетворяющая уравнению (4). 
ДоКАЗАТЕЛЬСТВо осуществляется по той же схеме, что и доказательство теоремы 1 работы [4], с некоторьм усложнением рассуждений. Действительно, согласно лемме 7 достаточно ограничиться рассмотрением уравнения

$$
y^{\prime \prime}+\left(a+\frac{Q_{1}}{Q_{2}}\right) y^{\prime}+\left(b+\frac{Q_{0}}{Q_{2}}\right) y=\frac{Q}{Q_{2}}, \quad(a, b) \neq(0,0),
$$

где $a, b \in \mathbb{A}, Q_{0}, Q_{1}, Q_{2}, Q \in \mathbb{A}[z], \max \left(\operatorname{deg} Q_{1}, \operatorname{deg} Q_{0}\right)<\operatorname{deg} Q_{2}$. Обозначим $\xi_{1}, \ldots, \xi_{m}$, $\xi_{0}=0$ все конечные особые точки уравнения (21). Пусть его наименьший показатель в точке $\xi_{j}, 1 \leqslant j \leqslant m$, равен $n_{j} \geqslant 0$, а $\lambda, \lambda_{1}-$ его показатели в точке $\xi_{0}=0$. Рассмотрим многочлен $P=\left(z-\xi_{1}\right)^{n_{1}} \cdots\left(z-\xi_{m}\right)^{n_{m}}$. Согласно леммам $2,4,5,9$ найдется многочлен $P_{1} \in \mathbb{A}[z]$ такой, что функция $g(z)=\left(f(z)-P_{1}\right) / P$ является $E$-функцией, удовлетворяющей дифференциальному уравнению вида (21), не имеющему особых точек, отличных от $\xi_{0}, \xi_{1}, \ldots, \xi_{m}$, причем всякая особая точка является регулярной. Из выбора многочлена $P$ следует, что наименьший показатель полученного дифференциального уравнения в каждой точке $\xi_{j} \neq 0$ равен нулю. Отсюда и из леммы 8 получаем, что функция $Q_{0} / Q_{2}$ в точке $\xi_{j} \neq 0$ может иметь полюсы не более, чем 1-го порядка. Тогда из леммы 3 следует, что функция $g(z)$ удовлетворяет либо дифференциальному уравнению (4), либо дифференциальному уравнению

$$
\begin{aligned}
y^{\prime \prime} & +\left(a+\frac{1-\lambda-\lambda_{1}}{z}-\frac{k_{1}}{z-\xi_{1}}-\cdots-\frac{k_{m}}{z-\xi_{m}}\right) y^{\prime} \\
& +\left(b+\frac{b_{0}}{z}+\frac{\lambda \lambda_{1}}{z^{2}}+\frac{b_{1}}{z-\xi_{1}}+\cdots+\frac{b_{m}}{z-\xi_{m}}\right) y=\frac{Q_{3}}{Q_{2}}
\end{aligned}
$$

где $a, \lambda, \lambda_{1}, b, b_{0}, b_{1}, \ldots, b_{m} \in \mathbb{A}, k_{j} \in \mathbb{N}, \xi_{j} \in \mathbb{A}, j=1, \ldots, m$.

Пусть $k=k_{1}+\cdots+k_{m}$. Дальнейшее доказательство теоремы будем проводить индукцией по $k$. Если $k=0$, то утверждение теоремы выполняется. Предположим, что утверждение справедливо для всех $E$-функций, удовлетворяющих произвольным дифференциальньм уравнениям вида (22) при любьх $m \in \mathbb{Z}^{+}, k \leqslant n-1, n \geqslant 1$, и докажем его для $k=n$. Если $\left(b_{m}, \lambda\right) \neq(0,0)$, рассмотрим функцию $g_{1}(z)=e^{\alpha_{1} z} z^{-\lambda} g(z)$, где $\alpha_{1}=\lambda / \xi_{m}-b_{m} / k_{m}$. Как следует из леммы $9, g_{1}(z)$ удовлетворяет дифференциальному уравнению

$$
\begin{aligned}
y^{\prime \prime} & +\left(a-2 \alpha_{1}+\frac{1-\lambda_{1}+\lambda}{z}-\frac{k_{1}}{z-\xi_{1}}-\cdots-\frac{k_{m}}{z-\xi_{m}}\right) y^{\prime} \\
& +\left(b^{*}+\frac{b_{0}^{*}}{z}+\frac{b_{1}^{*}}{z-\xi_{1}}+\cdots+\frac{b_{m-1}^{*}}{z-\xi_{m-1}}\right) y=e^{\alpha_{1} z} z^{-\lambda} \frac{Q_{3}}{Q_{2}}
\end{aligned}
$$

где $b^{*}, b_{0}^{*}, \ldots, b_{m-1}^{*} \in \mathbb{A}$, которое можно записать в виде

$$
y^{\prime \prime}+\frac{Q_{4}}{Q_{2}} y^{\prime}+\frac{Q_{5}}{Q_{2}} y=e^{\alpha_{1} z} z^{-\lambda} \frac{Q_{3}}{Q_{2}}
$$

где $Q_{4}, Q_{5} \in \mathbb{A}[z], \operatorname{deg} Q_{4} \leqslant m+1, Q_{2}=z\left(z-\xi_{1}\right) \cdots\left(z-\xi_{m}\right)$. 
Рассмотрим случай $Q_{5} \equiv 0$. Тогда решениями однородных дифференциальных уравнений, соответствующих уравнениям (23) и (22), являются функции $y_{*} \equiv 1$ и $v_{*}=$ $e^{-\alpha_{1} z} z^{\lambda}$ соответственно. Будем искать функцию $g(z)$ в виде

$$
g=v_{*} \int w d z
$$

Дифференцируя равенство (24), получаем

$$
g^{\prime}=\left(-\alpha_{1}+\frac{\lambda}{z}\right) g+v_{*} w
$$

откуда следует, что $z v_{*} w$ есть $E$-функция. Подставляя равенство $(24)$ в уравнение $(22)$, найдем уравнение, которому удовлетворяет функция $w$. Далее, воспользовавшись леммой 11, получим, что $E$-функция $z v_{*} w$ удовлетворяет уравнению

$$
y^{\prime}=\left(\alpha_{1}-a+\frac{\lambda_{1}}{z}+\frac{k_{1}}{z-\xi_{1}}+\cdots+\frac{k_{m}}{z-\xi_{m}}\right) y+\frac{z Q_{3}}{Q_{2}} .
$$

Согласно доказательству теоремы 2 работы [3] отсюда имеем

$$
z v_{*} w=P_{2} \varphi_{-\lambda_{1}}\left(\left(\alpha_{1}-a\right) z\right)+P_{3}, \quad P_{2}, P_{3} \in \mathbb{A}[z], \quad \lambda_{1} \in \mathbb{Q} .
$$

Из этого равенства и (25) следует, что функция $g(z)$ удовлетворяет уравнению (17), откуда ввиду леммы 13 получаем доказьваемое утверждение.

Если же в уравнении $(23) Q_{5} \not \equiv 0$, то $Q_{5}=z^{l_{0}}\left(z-\xi_{1}\right)^{l_{1}} \cdots\left(z-\xi_{m}\right)^{l_{m}} Q_{6}, Q_{6} \in \mathbb{A}[z]$, $l_{j} \geqslant 0, l_{m} \geqslant 1, \operatorname{deg} Q_{6}=\mu \leqslant m+1-\left(l_{0}+l_{1}+\cdots+l_{m}\right), Q_{6}=c\left(z-\beta_{1}\right)^{p_{1}} \cdots\left(z-\beta_{s}\right)^{p_{s}}$, $p_{i} \in \mathbb{N}, \beta_{i} \neq \beta_{j}, \beta_{i} \neq \xi_{j}, c \in \mathbb{A} \backslash\{0\}$.

Согласно лемме 10 функция $g_{1}^{\prime}(z)$ удовлетворяет уравнению

$$
\begin{aligned}
y^{\prime \prime} & +\left(a-2 \alpha_{1}+\frac{1-a_{0}-l_{0}}{z}-\sum_{j=1}^{m} \frac{k_{j}-1+l_{j}}{z-\xi_{j}}-\sum_{i=1}^{s} \frac{p_{i}}{z-\beta_{i}}\right) y^{\prime} \\
& +\left(\frac{a_{0} l_{0}}{z^{2}}+\sum_{j=1}^{m} \frac{k_{j} l_{j}}{\left(z-\xi_{j}\right)^{2}}+\frac{Q_{7}}{Q_{9}}\right) y=e^{\alpha_{1} z} z^{-\lambda-1} \frac{Q_{8}}{Q_{10}}
\end{aligned}
$$

где $Q_{7}, Q_{8}, Q_{10} \in \mathbb{A}[z], Q_{9}=\left(z-\beta_{1}\right) \cdots\left(z-\beta_{s}\right) Q_{2}, a_{0}=\lambda_{1}-\lambda-1$.

Функция $e^{-\alpha_{1} z} z^{\lambda+1} g_{1}^{\prime}(z)$ является $E$-функцией, удовлетворяющей дифференциальному уравнению

$$
\begin{aligned}
y^{\prime \prime} & +\left(a-\frac{\lambda_{1}+\lambda+l_{0}}{z}-\sum_{j=1}^{m} \frac{k_{j}-1+l_{j}}{z-\xi_{j}}-\sum_{i=1}^{s} \frac{p_{i}}{z-\beta_{i}}\right) y^{\prime} \\
& +\left(\frac{a_{1} \lambda_{1}}{z^{2}}+\sum_{j=1}^{m} \frac{k_{j} l_{j}}{\left(z-\xi_{j}\right)^{2}}+\frac{Q_{11}}{Q_{9}}\right) y=\frac{Q_{8}}{Q_{10}}
\end{aligned}
$$

где $Q_{11} \in \mathbb{A}[z], a_{1}=\lambda+l_{0}+1$. 
Показатели однородного дифференциального уравнения, соответствующего уравнению $(26)$, в точке $\xi_{j} \neq 0$ согласно лемме 8 равны $l_{j}$ и $k_{j}$. Рассмотрим многочлен

$$
P_{4}=\left(z-\xi_{1}\right)^{l_{1}^{*}} \ldots\left(z-\xi_{m}\right)^{l_{m}^{*}},
$$

где $l_{j}^{*}=\min \left(l_{j}, k_{j}\right), 1 \leqslant j \leqslant m, l_{m}^{*} \geqslant 1$. Согласно леммам 4,8 и 9 существует многочлен $P_{5} \in \mathbb{A}[z]$ такой, что функция $g_{2}(z)=\left(e^{-\alpha_{1} z} z^{\lambda+1} g_{1}^{\prime}(z)-P_{5}\right) / P_{4}$ является $E$-функцией, удовлетворяющей дифференциальному уравнению, коэффициент которого при $y$ в точках $\xi_{j} \neq 0$ имеет полюсы не более, чем 1-го порядка, а коэффициент при $y^{\prime}$ равен

$$
a-\frac{\lambda_{1}+\lambda+l_{0}}{z}-\sum_{j=1}^{m} \frac{k_{j}-1+l_{j}-2 l_{j}^{*}}{z-\xi_{j}}-\sum_{i=1}^{s} \frac{p_{i}}{z-\beta_{i}} .
$$

Величина $k$ у полученного дифференциального уравнения не превосходит

$$
n+\mu-m+\left(l_{1}+\cdots+l_{m}\right)-2\left(l_{1}^{*}+\cdots+l_{m}^{*}\right) \leqslant n+1-2\left(l_{1}^{*}+\cdots+l_{m}^{*}\right) \leqslant n-1 .
$$

Согласно индуктивному предположению функция $g_{2}(z)$ и, следовательно, $e^{-\alpha_{1} z} z^{\lambda+1} \times$ $g_{1}^{\prime}(z)$, имеет вид $(18)$ или $(16)$, а $g_{1}^{\prime}(z)$ имеет вид

$$
\left(R_{0} \varphi_{\lambda}(\alpha z)+R_{1} \varphi_{\lambda_{1}}\left(\alpha_{1} z\right)+R\right) e^{\alpha_{1} z} z^{-\lambda-1},
$$

либо

$$
\left(R_{0} f_{1}(z)+R_{1} f_{1}^{\prime}(z)+R\right) e^{\alpha_{1} z} z^{-\lambda-1},
$$

где $R_{0}, R_{1}, R \in \mathbb{A}(z), f_{1}(z)$ - $E$-функция, являющаяся решением уравнения (4). Но тогда из уравнения (23), которому удовлетворяет функция $g_{1}(z)$, получаем, что $g_{1}(z)$ имеет аналогичньй вид. Следовательно, $g(z)$ и $f(z)$ имеют вид (18) или (16). Лемма доказана.

3. Доказательство теоремы 2. Достаточность условий теоремы проверяется непосредственным вычислением (подробнее об этом см. п. 4, леммы 16 и 17).

Для дальнейшего доказательства заметим, что согласно лемме 14 функция $f(z)$ имеет вид (16) или (18). Из равенства (16) следует $f^{\prime}(z)=R_{2} f_{1}(z)+R_{3} f_{1}^{\prime}(z)+R_{4}$, где $R_{2}, R_{3}, R_{4} \in \mathbb{A}(z)$. Если $R_{1} R_{2}-R_{0} R_{3} \equiv 0$, то

$$
R_{1} f^{\prime}(z)-R_{3} f(z)=\left(R_{1} R_{2}-R_{0} R_{3}\right) f_{1}(z)+R_{1} R_{4}-R_{3} R \in \mathbb{A}(z)
$$

и, следовательно, $f(z)$ удовлетворяет линейному дифференциальному уравнению 1-го порядка с коэффициентами из $\mathbb{C}(z)$. Тогда согласно теореме 2 работы [3]

$$
f(z)=P \varphi_{\lambda}(\alpha z)+P_{1}
$$

где $P, P_{1} \in \mathbb{A}[z], \alpha \in \mathbb{A}, \lambda \in \mathbb{Q}$, и теорема справедлива. Если $R_{1} R_{2}-R_{0} R_{3} \not \equiv 0$, то функции $f_{1}(z), f_{1}^{\prime}(z)$ линейно выражаются через функции $f(z), f^{\prime}(z)$ и поэтому являются алгебраически зависимьми над $\mathbb{C}(z)$. Предположив, что функция $f_{1}(z)$ имеет вид (5) или (6), из равенства (16) получим, что функция $f(z)$ также имеет вид (5) (соответственно (6)), но уже с $P_{0}, P_{1}, P \in \mathbb{A}(z)$. Если $f(z)$ имеет вид $(18)$, то с помощью аналогичных рассуждений и теоремы 4 из [2, гл. 5], а также формулы

$$
\varphi_{\lambda}(z)=1+\sum_{n=1}^{l-1} \frac{z^{n}}{(\lambda+1) \cdots(\lambda+n)}+\frac{z^{l}}{(\lambda+1) \cdots(\lambda+l)} \varphi_{\lambda+l}(z),
$$

где $l \in \mathbb{N}$, снова убеждаемся, что имеют место равенства (5) или (6). Таким образом, для доказательства теоремы достаточно установить, что если $f(z)$ удовлетворяет уравнению (4), то она имеет вид (5) или (6) с $P_{0}, P_{1}, P \in \mathbb{A}(z)$, и что отсюда следуют включения $P_{0}, P_{1}, P \in \mathbb{A}[z]$. 
ЛЕмма 15. Если Е-функиия $f(z)$ удовлетворяет уравнению (4) и алгебраичес$\kappa и$ зависит $c f^{\prime}(z)$ над $\mathbb{C}(z)$, то существует решение $y_{*}$ однородного уравнения, соответствующего уравнению (4), такое, что $y_{*}^{\prime} / y_{*}=P / S, P, S \in \mathbb{C}[z]$.

ДокАЗАТЕЛЬСТво. Согласно лемме 10 книги [2, гл. 6] однородное уравнение, соответствующее уравнению (4), имеет решение $y_{*}$ такое, что его логарифмическая производная $y_{*}^{\prime} / y_{*}$ есть алгебраическая функция над $\mathbb{C}(z)$. Функция $v_{*}=\exp (a z / 2) y_{*}$ согласно следствию из леммы 9 удовлетворяет уравнению

$$
y^{\prime \prime}+\frac{a_{1}}{z} y^{\prime}+\left(b^{*}+\frac{b_{1}^{*}}{z}+\frac{b_{2}^{*}}{z^{2}}\right) y=0, \quad a_{1}, b^{*}, b_{1}^{*}, b_{2}^{*} \in \mathbb{A} .
$$

Рассмотрим функцию $u=v_{*}^{\prime} / v_{*}=y_{*}^{\prime} / y_{*}+a / 2$. Она является алгебраической и удовлетворяет уравнению Риккати

$$
u^{\prime}+u^{2}+\frac{a_{1}}{z} u+b^{*}+\frac{b_{1}^{*}}{z}+\frac{b_{2}^{*}}{z^{2}}=0 .
$$

Пусть $b^{*} \neq 0$. Докажем, что $z=\infty$ не является точкой ветвления функции $u$. Отсюда будет следовать, что эта функция не имеет точек ветвления вообще.

Разложим функцию $u$ в окрестности точки $z=\infty$ в степенной ряд с рациональньми показателями:

$$
u=\sum_{k=1}^{\infty} c_{k} z^{r_{k}}, \quad c_{1} \neq 0, \quad r_{1}>r_{2}>r_{3}>\cdots .
$$

Подставив разложение (30) в уравнение $(29)$, убеждаемся, что $r_{1}=0, c_{1}^{2}=-b^{*}$. Если не все показатели $r_{k}$ в ряде (30) целые, то пусть $r_{k_{1}}$ - наибольший дробньй показатель такой, что $c_{k_{1}} \neq 0$. Тогда, подставляя разложение $(30)$ в уравнение $(29)$ и рассматривая коэффищиент при наибольшей дробной степени $z$, получаем $c_{1} c_{k_{1}}=0$, что противоречит нашим допущениям. Следовательно, $u \in \mathbb{C}(z)$.

Пусть теперь в уравнении $(28) b^{*}=0$. Обозначив $\lambda_{1}$ и $\lambda_{2}$ показатели этого уравнения в точке $z=0$, ввиду леммы 8 получим $a_{1}=1-\lambda_{1}-\lambda_{2}, b_{2}^{*}=\lambda_{1} \lambda_{2}$. Функция $v_{* *}=z^{-\lambda_{1}} v_{*}$ согласно лемме 9 удовлетворяет уравнению

$$
y^{\prime \prime}+\frac{1-l}{z} y^{\prime}+\frac{\alpha}{z} y=0,
$$

где $l=\lambda_{2}-\lambda_{1}, \alpha=b_{1}^{*}$.

Если $\alpha=0$, то уравнению (31) удовлетворяет функция $y \equiv 1$, а однородному уравнению, соответствующему (4), - функция $y_{*}=z^{\lambda_{1}} e^{-a z / 2}$, откуда снова получаем $y_{*}^{\prime} / y_{*} \in$ $\mathbb{C}(z)$. Если $\alpha \neq 0$, то алгебраическая функция $u=v_{* *}^{\prime} / v_{* *}=-\lambda / z+a / 2+y_{*}^{\prime} / y_{*}$ удовлетворяет уравнению Риккати

$$
u^{\prime}+u^{2}+\frac{1-l}{z} u+\frac{\alpha}{z}=0 .
$$

Подставляя разложение (30) в уравнение $(32)$, убеждаемся, что $r_{1}=-1 / 2, c_{1}= \pm \sqrt{-\alpha}$, $r_{2}=-1, c_{2}=l / 2-1 / 4$. Докажем, что знаменатели рациональных показателей в ряде $(30)$ не могут быть больше, чем 2 . Если это не так, то пусть $r_{k_{1}}$ - наибольший показатель со знаменателем, содержащим простой множитель $p>2$ или множитель $2^{n}$, 
$n>1$, a $c_{k_{1}} \neq 0$. Тогда, подставив разложение (30) в уравнение $(32)$, получим, что наибольший показатель со знаменателем, содержащим аналогичньй множитель, равен $-1 / 2+r_{k_{1}}$, а коэффициент при степени $z$ с таким показателем равен $2 c_{1} c_{k_{1}} \neq 0$. Полученное противоречие доказьвает, что наибольший общий знаменатель показателей $r_{1}, r_{2}, r_{3}, \ldots$ равен 2 .

Разложим функцию $u$ в окрестности точки $z=0$ :

$$
u=\sum_{k=1}^{\infty} d_{k} z^{t_{k}}, \quad d_{1} \neq 0, \quad t_{1}<t_{2}<t_{3}<\cdots
$$

Подставив разложение (33) в уравнение (32), убеждаемся, что $-1 \leqslant t_{1} \leqslant 0$, причем если $t_{1}=-1$, то $d_{1}=l$. Поскольку порядки ветвления в точках $z=0$ и $z=\infty$, очевидно, совпадают, наибольший общий знаменатель показателей $t_{1}, t_{2}, t_{3}, \ldots$ также равен 2 . Обозначим $t_{k_{1}}$ наименьший показатель вида $m+1 / 2$, где $m \in \mathbb{Z}$. Подставляя разложение $(33)$ в уравнение $(32)$, в случае $t_{1} \neq-1$ получим, что $(m+3 / 2-l) d_{k_{1}}=0$, а в случае $t_{1}=-1-(m+3 / 2+l) d_{k_{1}}=0$, откуда $l$ должно равняться половине нечетного числа. Показатели дифференциального уравнения (31) в точке $z=0$ есть числа $l$ и 0 . Следовательно, фундаментальную систему решений этого уравнения образуют функции $f_{1}(z)$ и $z^{l} f_{2}(z)$, где $f_{1}(z), f_{2}(z)$ - некоторые целые функции, определяемые однозначно с точностью до множителя из $\mathbb{C}, f_{1}(0) f_{2}(0) \neq 0$. Нетрудно проверить, что уравнению $(31)$ удовлетворяют функции

$$
\begin{aligned}
K_{-l}(2 \sqrt{\alpha z}) & =\sum_{n=0}^{\infty} \frac{(-1)^{n} \alpha^{n}}{n !(1-l) \cdots(n-l)} z^{n} \\
z^{l} K_{l}(2 \sqrt{\alpha z}) & =z^{l} \sum_{n=0}^{\infty} \frac{(-1)^{n} \alpha^{n}}{n !(l+1) \cdots(l+n)} z^{n}
\end{aligned}
$$

где $K_{\lambda}(z)$ - функция Зигеля (см. [2, с. 18, 19]). Однородное уравнение, соответствующее исходному уравнению (4), имеет фундаментальную систему решений $e^{-a z / 2} z^{\lambda_{1}} f_{1}(z)$, $e^{-a z / 2} z^{l+\lambda_{1}} f_{2}(z)$. Эти функции вместе с исходной функцией $f(z)$ образуют фундаментальную систему решений однородного дифференциального уравнения 3-го порядка (8), где $m=2$. Но согласно доказанному Андрэ (см. [8, следствие 4.4]) фундаментальную систему решений в нашем случае должны образовьвать функции $f(z), z^{\alpha_{1}} f_{3}(z)$, $z^{\alpha_{2}} f_{4}(z)$, где $\alpha_{1}, \alpha_{2} \in \mathbb{Q}, f_{3}(z), f_{4}(z)-E$-функции. Тогда $e^{-a z / 2} f_{1}(z)$ и $e^{-a z / 2} f_{2}(z)$ должны быть $E$-функциями, что при $\alpha \neq 0$ невозможно. Лемма доказана.

Записав функцию $u$ в виде суммы простейших дробей и подставив еев уравнение (29), приходим к выводу, что

$$
\frac{y_{*}^{\prime}}{y_{*}}=\frac{P}{S}=b_{0}+\frac{b}{z}+\sum_{j=1}^{l} \frac{1}{z-\alpha_{j}}, \quad b_{0}, b \in \mathbb{A}, \quad S=z\left(z-\alpha_{1}\right) \cdots\left(z-\alpha_{l}\right), \quad \alpha_{1}, \ldots, \alpha_{l} \in \mathbb{C} .
$$

Представим функцию $f$ в виде

$$
f=y_{*} \int w d z
$$


Дифференцируя равенство (34), получаем

$$
f^{\prime}=\frac{P}{S} f+y_{*} w
$$

откуда следует, что $y_{*} w S$ есть целая функция. Подставив равенство $(34)$ в уравнение (4) и воспользовавшись леммой 11 , получим, что функция $y_{*} w S$ удовлетворяет уравнению $y^{\prime}=\left(S^{\prime} / S-a-a_{1} / z-P / S\right) y+\left(c+c_{1} / z\right) S$, равносильному

$$
y^{\prime}=\left(a^{*}+\frac{a_{1}^{*}}{z}\right) y+\left(c+\frac{c_{1}}{z}\right) S, \quad a^{*}, a_{1}^{*}, c, c_{1} \in \mathbb{A} \text {. }
$$

Следовательно, $y_{*} w S=b_{1} \varphi_{\lambda}(\alpha z)+P_{1}$, где $\alpha, \lambda \in \mathbb{A}, b_{1} \in \mathbb{C}, P_{1} \in \mathbb{C}[z]$ (см. [3, доказательство теоремы 2]). Отсюда и из уравнения (35) получим, что $E$-функция $f(z)$ удовлетворяет уравнению

$$
y^{\prime}=\frac{P}{S} y+\frac{b_{1} \varphi_{\lambda}(\alpha z)+P_{1}}{S} .
$$

Согласно результатам книги $[2$, гл. $3, \S 2] b_{1} \in \mathbb{A}, P, P_{1}, S \in \mathbb{A}[z]$. Тогда ввиду уравнения (35) $y_{*} w S$ есть $E$-функция, а $\lambda \in \mathbb{Q}$.

Из равенства (36) и условий теоремы следует, что функции $f(z)$ и $\varphi_{\lambda}(\alpha z)$ алгебраически зависимы над $\mathbb{C}(z)$. Пусть $G(x)=x^{r}+u_{r-1} x^{r-1}+\cdots+u_{1} x+u_{0}$ - минимальный многочлен функции $f(z)$ над полем $\mathbf{L}=\mathbb{C}\left(z, \varphi_{\lambda}(\alpha z)\right)$. Сопряженные над $\mathbf{L} \mathrm{c} f(z)$ функции $f_{1}=f(z), f_{2}, \ldots, f_{r}$ удовлетворяют уравнению (36) (см. [2, гл. 9, следствие из леммы 2]). Следовательно, $f_{2}=f_{1}+c_{2} \psi, \ldots, f_{r}=f_{1}+c_{r} \psi$, где $\psi=e^{b_{0} z} z^{b-1} S$ - решение однородного уравнения $y^{\prime}=(P / S) y, c_{i} \neq c_{j} \neq 0$ при $i \neq j$. Подставив полученные выражения для функций $f_{j}, j=2, \ldots, r$, в тождество $G\left(f_{j}\right)-G\left(f_{1}\right)=0$, имеем

$$
\begin{aligned}
r f_{1}^{r-1} c_{j} \psi & +\cdots+\left(c_{j} \psi\right)^{r}+\left((r-1) f_{1}^{r-2} c_{j} \psi+\cdots+\left(c_{j} \psi\right)^{r-1}\right) u_{r-1}+\cdots \\
& +\left(2 f_{1} c_{j} \psi+\left(c_{j} \psi\right)^{2}\right) u_{2}+c_{j} \psi u_{1}=0, \quad j=2,3, \ldots, r
\end{aligned}
$$

что равносильно

$$
\begin{gathered}
\left(c_{j} \psi\right)^{r-1}+\left(c_{j} \psi\right)^{r-2} v_{r-2}+\cdots+c_{j} \psi v_{1}+v_{0}=0, \quad j=2,3, \ldots, r \\
v_{k} \in \mathbf{L}\left[f_{1}\right], \quad k=0,1, \ldots, r-2, \quad v_{r-2}=r f_{1}+u_{r-1} .
\end{gathered}
$$

При $r=2$ отсюда имеем $c_{2} \psi+2 f_{1}+u_{1}=0$. Если $r \geqslant 3$, то полученные равенства можно рассматривать как систему линейных неоднородных алгебраических уравнений относительно функций $\psi^{r-1}, \psi^{r-2} v_{r-2}, \ldots, \psi v_{1}$, определитель матрицы коэффициентов которой есть определитель Вандермонда $V=V\left(c_{2}, \ldots, c_{r}\right) \neq 0$, умноженньй на $c_{2} \cdots c_{r} \neq 0$. Согласно правилу Крамера

$$
\psi^{r-1} D=-v_{0}\left|\begin{array}{cccc}
1 & c_{2}^{r-2} & \ldots & c_{2} \\
\vdots & \vdots & \ddots & \vdots \\
1 & c_{r}^{r-2} & \ldots & c_{r}
\end{array}\right|, \quad \psi^{r-2} v_{r-2} D=-v_{0}\left|\begin{array}{cccc}
c_{2}^{r-1} & 1 & \ldots & c_{2} \\
\vdots & \vdots & \ddots & \vdots \\
c_{r}^{r-1} & 1 & \ldots & c_{r}
\end{array}\right|
$$

где $D=c_{2} \cdots c_{r} V$, откуда $\psi=c_{*} v_{r-2}=c_{*}\left(r f_{1}+u_{r-1}\right), c_{*} \in \mathbb{C}$. Таким образом, при любом $r \in \mathbb{N} f(z)=c^{*} e^{b_{0} z} z^{b-1} S+u^{*}$, где $c^{*} \in \mathbb{C}, u^{*} \in \mathbf{L}$. Ввиду алгебраической зависимости функций $f(z)$ и $\varphi_{\lambda}(\alpha z)$ это возможно лишь в следующих случаях:

1) $\lambda \notin \mathbb{Z}^{+}, c^{*}=0, f(z) \in \mathbf{L}$

2) $\lambda=k \in \mathbb{Z}^{+}, \alpha=\sigma b_{0}, \sigma \in \mathbb{Q}, f(z) \in \mathbb{C}\left(z, e^{\alpha_{*} z}\right), \alpha_{*} \in \mathbb{A}($ см. [2, гл. 5, лемма 6]). 
Рассмотрим случай 1$)$. Пусть $f(z)=A / B$, где

$$
A=A_{k} \varphi_{\lambda}^{k}(\alpha z)+\cdots+A_{1} \varphi_{\lambda}(\alpha z)+A_{0}, \quad B=B_{n} \varphi_{\lambda}^{n}(\alpha z)+\cdots+B_{1} \varphi_{\lambda}(\alpha z)+B_{0},
$$

$A_{0}, \ldots, A_{k}, B_{0}, \ldots, B_{n} \in \mathbb{C}[z], k, n \in \mathbb{Z}^{+}$. Докажем, что $n=0$. Дифференцируя равенство $B f=A$, с учетом (36) получим

$$
\left(B^{\prime}+\frac{P}{S} B\right) f=A^{\prime}-\frac{\left(b_{1} \varphi_{\lambda}(\alpha z)+P_{1}\right) B}{S} .
$$

Поскольку степень многочлена $B^{\prime}+P B / S$ относительно $\varphi_{\lambda}(\alpha z)$ не превосходит степени $B$, эти многочлены отличаются друг от друга множителем из $\mathbb{C}[z]$. Предположим, что $n \geqslant 1$. Тогда

$$
\begin{aligned}
\frac{B_{n}^{\prime}+n(\alpha-\lambda / z) B_{n}+(P / S) B_{n}}{B_{n}} \\
\quad=\frac{B_{n-1}^{\prime}+(n-1)(\alpha-\lambda / z) B_{n-1}+(P / S) B_{n-1}+(n \lambda / z) B_{n}}{B_{n-1}},
\end{aligned}
$$

что равносильно равенству

$$
\frac{B_{n}^{\prime}}{B_{n}}+\alpha-\frac{\lambda}{z}=\frac{B_{n-1}^{\prime}}{B_{n-1}}+\frac{n \lambda B_{n}}{z B_{n-1}}
$$

Пусть многочлены $B_{n}$ и $B_{n-1}$ имеют число 0 корнем кратности соответственно $l \geqslant 0$ и $l_{1} \geqslant 0$. Так как в разложении на простейшие дроби левой (а следовательно, и правой) части равенства (38) могут присутствовать только дроби с линейными функциями в знаменателе, то $l_{1} \leqslant l$. Если $l_{1}<l$, то $\lambda \in \mathbb{N}$, что противоречит условию. Поэтому $l_{1}=l$. Если $\xi \neq 0$ - корень многочлена $B_{n}$, то из равенства (38) следует, что он должен быть также корнем многочлена $B_{n-1}$ не меньшей кратности. Но тогда $\operatorname{deg} B_{n} \leqslant \operatorname{deg} B_{n-1}$ и из (38) получаем, что $\alpha=0, f(z) \in \mathbb{C}[z]$. Таким образом, далее можно считать, что $n=0, B \in \mathbb{C}[z]$.

Допустим, что $k \geqslant 3$. Тогда из равенства (37) получим

$$
\frac{A_{k}^{\prime}+k(\alpha-\lambda / z) A_{k}}{A_{k}}=\frac{A_{k-1}^{\prime}+(k-1)(\alpha-\lambda / z) A_{k-1}+(k \lambda / z) A_{k}}{A_{k-1}}
$$

что равносильно равенству

$$
\frac{A_{k}^{\prime}}{A_{k}}+\alpha-\frac{\lambda}{z}=\frac{A_{k-1}^{\prime}}{A_{k-1}}+\frac{k \lambda A_{k}}{z A_{k-1}}
$$

Поскольку полученное равенство полностью аналогично (38), делаем вьвод, что $k \leqslant 2$,

$$
f(z)=\frac{P_{2} \varphi_{\lambda}^{2}(\alpha z)+P_{3} \varphi_{\lambda}(\alpha z)+P_{4}}{P_{5}}
$$

где $P_{2}, \ldots, P_{5}$ - взаимно простые многочлены из $\mathbb{C}[z]$. Можно считать, что $P_{2}, \ldots, P_{5} \in$ $\mathbb{A}[z]$ (см. $\left[2\right.$, гл. 3, лемма 2]). Многочлен $P_{5}$ не может иметь корнем число $\xi \in \mathbb{A} \backslash\{0\}$, 
так как отсюда следовало бы, что $\varphi_{\lambda}(\alpha \xi) \in \mathbb{A}$. Поэтому $P_{5}=z^{l}, l \in \mathbb{Z}^{+}$. Но тогда, выражая $\varphi_{\lambda}(\alpha z)$ через $\varphi_{\lambda+l}(\alpha z)$ по формуле $(27)$, получаем, что многочлены в числителе преобразованной правой части равенства (39) также делятся на $z^{l}$. Следовательно, в случае 1) утверждение теоремы справедливо.

Случай 2) рассматривается аналогично случаю 1) с той лишь разницей, что при $\lambda=0$ к противоречию должны приводиться утверждения, что многочлен $B$ содержит хотя бы два члена различных степеней относительно $e^{\alpha_{*} z}=\varphi_{0}\left(\alpha_{*} z\right)$, а многочлен $A-$ более двух таких членов различных ненулевых степеней. В результате вместо равенства (39) мы получим равенство

$$
f(z)=\frac{P_{2} \varphi_{0}\left(\alpha_{*} l z\right)+P_{3} \varphi_{0}\left(\alpha_{*} s z\right)+P_{4}}{P_{5}}, \quad P_{2}, \ldots, P_{5} \in \mathbb{A}[z], \quad l, s \in \mathbb{Z},
$$

и, повторяя оставшиеся рассуждения в доказательстве случая 1), завершаем доказательство теоремы.

4. Доказательство теоремы 3. Если функции $f(z)$ и $f^{\prime}(z)$ алгебраически независимы над $\mathbb{C}(z)$, то утверждение теоремы 3 следует из 2-й основной теоремы Шидловского [2, гл. 3]. Если же они алгебраически зависимы, то при условиях теоремы функция $f(z)$ имеет вид (5), где $\sigma \alpha P_{0} P_{1} \not \equiv 0, \sigma \neq 1$, либо вид (6), где $\alpha P_{0} \not \equiv 0$. Непосредственньм вычислением можно найти дифференциальные уравнения, которым удовлетворяет такая функция и установить следуюшие две леммы.

ЛЕмма 16. Функиия $f(z)$, имеющая вид (5), удовлетворяет уравнениям

$$
\begin{gathered}
y^{\prime}=\left(P_{0}^{\prime}+\left(\alpha-\frac{k}{z}\right) P_{0}\right) \varphi_{k}(\alpha z)+\left(P_{1}^{\prime}+\left(\sigma \alpha-\frac{k}{z}\right) P_{1}\right) \varphi_{k}(\sigma \alpha z)+\frac{k}{z} P_{0}+\frac{k}{z} P_{1}+P^{\prime} \\
y^{\prime}=\left(\alpha-\frac{k}{z}+\frac{P_{0}^{\prime}}{P_{0}}\right) y+T \varphi_{k}(\sigma \alpha z)+R \\
y^{\prime \prime}=\left((\sigma+1) \alpha-\frac{2 k}{z}+\frac{P_{0}^{\prime}}{P_{0}}+\frac{T^{\prime}}{T}\right) y^{\prime}+R_{1} y+R_{2}
\end{gathered}
$$

əəe

$$
T=P_{1}^{\prime}+\left((\sigma-1) \alpha-\frac{P_{0}^{\prime}}{P_{0}}\right) P_{1}, \quad R, R_{1}, R_{2} \in \mathbb{A}(z)
$$

Лемма 17. Функиия $f(z)$, имеющая вид (6), удовлетворяет уравнениям

$$
\begin{gathered}
y^{\prime}=\left(P_{0}^{\prime}+2\left(\alpha-\frac{\lambda}{z}\right) P_{0}\right) \varphi_{\lambda}^{2}(\alpha z)+\left(P_{1}^{\prime}+\left(\alpha-\frac{\lambda}{z}\right) P_{1}+\frac{2 \lambda}{z} P_{0}\right) \varphi_{\lambda}(\alpha z)+\frac{\lambda}{z} P_{1}+P^{\prime}, \\
y^{\prime}=\left(2\left(\alpha-\frac{\lambda}{z}\right)+\frac{P_{0}^{\prime}}{P_{0}}\right) y+T \varphi_{\lambda}(\alpha z)+R, \\
y^{\prime \prime}=\left(3\left(\alpha-\frac{\lambda}{z}\right)+\frac{P_{0}^{\prime}}{P_{0}}+\frac{T^{\prime}}{T}\right) y^{\prime}+R_{1} y+R_{2},
\end{gathered}
$$

əəe

$$
T=P_{1}^{\prime}+\left(-\alpha+\frac{\lambda}{z}-\frac{P_{0}^{\prime}}{P_{0}}\right) P_{1}+\frac{2 \lambda}{z} P_{0}, \quad R, R_{1} R_{2} \in \mathbb{A}(z) .
$$


Легко видеть, что при условиях теоремы 3 рациональные функции, являющиеся коэффициентами дифференциальных уравнений (40)-(43), определены однозначно. Докажем, что всякая точка $\xi \neq 0$, для которой числа $f(\xi), f^{\prime}(\xi)$ и 1 являются линейно зависимыми над $\mathbb{A}$, есть особая точка уравнения (1). Действительно, в силу линейной независимости значений функций $\varphi_{k}(\alpha z), \varphi_{k}(\sigma \alpha z)$ (соответственно $\left.\varphi_{\lambda}(\alpha z), \varphi_{\lambda}^{2}(\alpha z)\right)$ и 1 над $\mathbb{A}$ из равенств $(5),(6),(40),(42)$ следует, что числа $f(\xi), f^{\prime}(\xi)$ и 1 линейно зависимы тогда и только тогда, когда вектор $\left\{P_{0}(\xi), P_{1}(\xi)\right\}$ коллинеарен соответственно векторам

$$
\begin{gathered}
\left\{P_{0}^{\prime}(\xi)+\left(\alpha-\frac{k}{\xi}\right) P_{0}(\xi), P_{1}^{\prime}(\xi)+\left(\sigma \alpha-\frac{k}{\xi}\right) P_{1}(\xi)\right\} \\
\left\{P_{0}^{\prime}(\xi)+2\left(\alpha-\frac{\lambda}{\xi}\right) P_{0}(\xi), P_{1}^{\prime}(\xi)+\left(\alpha-\frac{\lambda}{\xi}\right) P_{1}(\xi)+\frac{2 \lambda}{\xi} P_{0}(\xi)\right\} .
\end{gathered}
$$

Если $P_{0}(\xi)=0, P_{1}(\xi) \neq 0$, то отсюда $P_{0}^{\prime}(\xi)=0$ и, следовательно, $\xi$ есть нуль функции $P_{0}$ кратности не меньше, чем 2 . Но тогда $\xi$ - полюс 1-го порядка функции $T$ и коэффициента при $y^{\prime}$ в уравнении (41) (соответственно (43)). Случай $P_{1}(\xi)=0, P_{0}(\xi) \neq 0$ для функции $f(z)$ вида (5) разбирается аналогично, а для функции вида (6) в силу пропорциональности векторов $\left\{P_{0}(\xi), P_{1}(\xi)\right\}$ и $(45)$ имеем $P_{1}^{\prime}(\xi)+(2 \lambda / \xi) P_{0}(\xi)=0$. Но тогда $\xi$ - нуль функции $T$ и полюс 1-го порядка коэффициента при $y^{\prime}$ в уравнении (43). Если $P_{0}(\xi) P_{1}(\xi) \neq 0$, то в силу пропорциональности векторов $\left\{P_{0}(\xi), P_{1}(\xi)\right\}$ и $(44)$ (соответственно (45)) имеем соответственно

$$
\begin{array}{r}
\frac{P_{1}^{\prime}(\xi)}{P_{1}(\xi)}-\frac{P_{0}^{\prime}(\xi)}{P_{0}(\xi)}+(\sigma-1) \alpha=0, \\
\frac{P_{1}^{\prime}(\xi)}{P_{1}(\xi)}-\frac{P_{0}^{\prime}(\xi)}{P_{0}(\xi)}+\frac{2 \lambda}{\xi} \frac{P_{0}(\xi)}{P_{1}(\xi)}-\alpha+\frac{\lambda}{\xi}=0 .
\end{array}
$$

Отсюда $T(\xi)=0$ и точка $\xi$ снова является полюсом 1-го порядка коэффициента при $y^{\prime}$. Если, наконец, $P_{0}(\xi)=P_{1}(\xi)=0$, то $\xi$ - неособая точка функции $T$ и вновь полюс 1 -го порядка коэффициента при $y^{\prime}$. Теорема доказана.

5. Доказательство теоремы 1. Достаточность условий теоремы следует из того, что если $E$-функция $f_{1}(z)$ удовлетворяет уравнению (4), то функция $f(z)$, определяемая равенством (3) или $(2)$, является $E$-функцией, линейно зависимой с $f^{\prime}(z), f^{\prime \prime}(z)$ и 1 над $\mathbb{C}(z)$.

Для доказательства необходимости заметим, что согласно лемме 14 всякая $E$-функция, удовлетворяющая уравнению (1), имеет вид (18) либо

$$
f(z)=\frac{P_{0} f_{1}(z)+P_{1} f_{1}^{\prime}(z)+P_{2}}{P_{3}},
$$

где $P_{0}, \ldots, P_{3}$ - взаимно простые многочлены из $\mathbb{A}[z], f_{1}(z)-E$-функщия, удовлетворяющая уравнению (4). Если функции $f_{1}(z), f_{1}^{\prime}(z)$ и 1 линейно зависимы над $\mathbb{C}(z)$, то функция $f(z)$ удовлетворяет линейному дифференциальному уравнению 1-го порядка с коэффициентами из $\mathbb{C}(z)$, и доказьваемая теорема следует из теоремы 2 работы [3]. Предположим, что функции $f_{1}(z), f_{1}^{\prime}(z)$ и 1 линейно независимы над $\mathbb{C}(z)$. Многочлен $P_{3}$ не может иметь корнем число $\xi \in \mathbb{A} \backslash\{0\}$, так как отсюда следовала бы линейная зависимость 
чисел $f(\xi), f^{\prime}(\xi)$ и 1 над $\mathbb{A}$, что противоречит теореме 3 . Поэтому $P_{3}=z^{l}, l \in \mathbb{Z}^{+}$. Рассмотрим многочлен $P_{4} \in \mathbb{A}[z], \operatorname{deg} P_{4} \leqslant l$, такой, что функция $g(z)=\left(f_{1}(z)-P_{4}\right) / z^{l+1}$ является целой и, следовательно, $E$-функцией. Тогда $f_{1}(z)=z^{l+1} g(z)+P_{4}$ и, подставляя это выражение в (46), получим $f(z)=P_{5} g(z)+P_{6} g^{\prime}(z)+P_{7}$, где $P_{5}, P_{6}, P_{7} \in \mathbb{A}[z]$. Повторяя конец доказательства леммы 12 , устанавливаем, что функция $g(z)$ либо $g(z)+c$, где $c \in \mathbb{A}$, удовлетворяет уравнению (4). Если же $f(z)$ имеет вид (18), то рассуждения проводятся аналогично, с использованием в случае $P_{3}=z^{l}$ равенства (27). Теорема доказана.

\section{СПИСОК ЦИТИРОВАННОЙ ЛИТЕРАТУРЫ}

[1] Siegel C.L. Über einige Anwendungen diophantischer Approximationen // Abh. Preuss. Acad. Wiss. Phys. -Math. Kl. 1929-1930. № 1. P. 1-70.

[2] Шидловский А. Б. Трансцендентные числа. М.: Наука, 1987.

[3] Горелов В.А. Об алгебраической независимости значений $E$-функций в особых точках и гипотезе Зигеля // Матем. заметки. 2000. Т. 67. № 2. С. 174-190.

[4] Горелов В.А. О гипотезе Зигеля для случая линейных однородных дифференциальных уравнений 2-го порядка // Матем. заметки. 2004. Т. 75. № 4. С. 549-565.

[5] Шидловский А. Б. О линейной независимости значений $E$-функций в алгебраических точках // Матем. заметки. 1994. Т. 55. № 2. С. 174-185.

[6] Камке Э. Справочник по обыкновенным дифференциальным уравнениям. М.: Наука, 1971.

[7] Айнс Э. Л. Обыкновенные дифференциальные уравнения. Харьков, 1939.

[8] André Y. Séries Gevrey de type arithmétique // Ann. of Math. 2000. V. 151. P. 705-756.

[9] Perron O. Über lineare Differentialgleichungen mit rationalen Koeffizienten // Acta Math. 1911. V. 34. P. 139-163.

[10] Валирон ЖК. Аналитические функции. М.: Гостехиздат, 1957.

Московский энергетический институт (технический университет)

Поступило E-mail: GorelovVA@mpei.ru

03.06.2004 